\title{
Virtual Mass of Any Shape of Body by using General Mapping Function
}

\author{
Norio ARAI ${ }^{1}$ \\ ${ }^{1}$ Department of Mechanical Precision Engineering, MJIIT, Universiti Teknologi Malaysia, Kuala Lumpur, 54100 Malaysia
}

\begin{abstract}
When an object is moving in acceleration with fluid interaction from surrounding, it possesses virtual mass (added mass) in which the total mass of object is higher than the original mass. The exact conformal mapping method was proposed to determine the virtual mass. However, it is difficult to determine the virtual mass for the body of random shape by using conformal mapping. Therefore the new concept of mapping function is proposed. It is based on Laurent series that has been proposed to map the body of random shape to a circle. The function of a body shape is expressed in Fourier series which is essential to determine the complex coefficients of mapping function (Laurent series). Proposed method is compared with exact conformal method (equilateral polygon) and the validity is confirmed.
\end{abstract}

\section{Introduction}

When the body is accelerated by fluid for instance, the body motion is expressed by the equation of motion like $m \ddot{x}+c \dot{x}+k x=f(t)$. Here $m$ (mass) is composed by both the pure mass of body and the 'virtual' mass. Especially, when the acceleration is large or the mass ratio is almost larger than unity, the effect of virtual mass should not be ignored. Although the virtual mass effect is very important term in motion, a little special cases could have been investigated because of the difficulty of calculating it analytically and/or exactly. While special cases are gotten from the famous text (Lamb[1], MilneThomson[2], Landau and Lifshitz[3]), the body geometries are restricted in the flat plate, circle, ellipse, and so on.

Here we need to specify the conformal mapping function from the target geometry ( $z$-plane) to the circle ( $\zeta$-plane), while it is very difficult to specify a general function for the conformal mapping analytically.

In this article the objective is to show the virtual mass of the any shape of body which geometry is used in architecture sometimes.

The conformal mapping function is given by the infinite series like Laurent Series $\sum \frac{a_{n}}{z^{n}}$, in which the body shape is expressed by using Fourier Series. Finally in order to validate the present method, some examples are shown.

\section{Analysys}

\subsection{Virtual mass and kinetic energy}

\footnotetext{
a Corresponding author: arai@utm.my or arai@cc.tuat.ac.jp
}

The flow field around a body at angle of attack ' $\alpha$ ' is expressed by using complex velocity potential ' $f$ ' as follows,

$$
f=U z e^{-i \alpha}+w
$$

where $U$ : speed of uniform flow, $w$ : 'disturbance' from 'body'.

For example, the flow around a circle is expressed

$$
\begin{aligned}
f & =U\left(z e^{-i \alpha}+\frac{a^{2}}{z} e^{i \alpha}\right) \\
& =U z e^{-i \alpha}+w \\
& ={ }^{\prime} \text { Uniform flow' }{ }^{\prime} \text { Disturbance' }^{\prime}
\end{aligned}
$$

As shown in Milne-Thomson[2], the total kinetic energy is derived by using 'disturbance $w$ ' as follows,

$$
T=\frac{1}{2} \rho \oint_{s} \frac{\partial}{\partial z}\left(w \frac{d \bar{w}}{d z}\right) d S
$$
Rule'

This equation is reduced to Eq.(6) by using 'Area

$T=-\frac{1}{4} i \rho \oint_{c 1} w d \bar{w}+\frac{1}{4} i \rho \oint_{c 2} w d \bar{w}$

where $c 1:$ inner boundary, $c 2:$ outer boundary.

When $c 2$ tends to infinity, the second term is vanished, then

$$
\begin{gathered}
\begin{array}{c}
T=-\frac{1}{4} i \rho \oint_{c 1} w d \bar{w}=\frac{1}{2} U^{2} M^{\prime} \\
=
\end{array} \\
\text { where } M^{\prime} \text { : votal Kintual mass. }
\end{gathered}
$$


Therfore, we can calculate the virtual mass by using $\mathrm{Eq}(7)$.

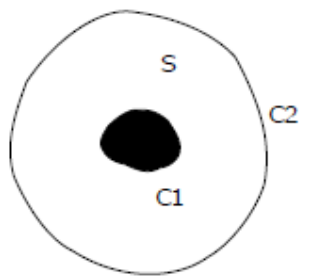

Figure 1. Flow field $S$, inner and outer boundaries $C 1, C 2$.

\subsection{General mapping function}

The body shape is expressed by using Fourier series as follows

$$
\text { real part } x_{n}=x_{n}(\theta)
$$

imaginary part $y_{n}=y_{n}(\theta)$

$x_{n}(\theta), y_{n}(\theta)=\frac{1}{2} a_{0}+\sum_{n=1}^{\infty}\left\{a_{n} \cos \left(\frac{n \pi \theta}{l}\right)+b_{n} \sin \left(\frac{n \pi \theta}{l}\right)\right\}$

While the body shape is expressed by Eq.(8), the general mapping function is defined (assumed) by the considerations in fluid dynamics as follows

$$
\begin{aligned}
z= & \sum_{n=0}^{\infty} A_{n} \zeta^{n}+\sum_{n=1}^{\infty} \frac{B_{n}}{\zeta^{n}} \\
= & A_{0}+A_{1} \zeta+A_{2} \zeta^{2}+A_{3} \zeta^{3}+\cdots \cdots \\
& +\frac{B_{1}}{\zeta}+\frac{B_{2}}{\zeta^{2}}+\frac{B_{3}}{\zeta^{3}}+\cdots \cdots
\end{aligned}
$$

where $\quad A_{n}={ }^{\mathrm{R}} A_{n}+i^{\mathrm{I}} A_{n} \quad(n=0,1,2,3, \cdots)$

$$
B_{n}={ }^{\mathrm{R}} B_{n}+i{ }^{\mathrm{I}} B_{n} \quad(n=1,2,3, \cdots)
$$

The body shape (Fourier series) is compared with the mapping function $(z)$. Then,

$$
\begin{array}{ll}
n=0 \quad: \quad & { }^{\mathrm{R}} A_{0}=\frac{1}{2} x a_{0}, \quad{ }^{\mathrm{I}} A_{0}=\frac{1}{2} y a_{0} \\
\vdots & \\
n=n \quad: \quad & a^{n \mathrm{R}} A_{n}+\frac{1}{a^{n}}{ }^{\mathrm{R}} B_{n}={ }^{x} a_{n}, \quad-a^{n \mathrm{I}} A_{n}+\frac{1}{a^{n}}{ }^{\mathrm{I}} B_{n}={ }^{x} b_{n} \\
& a^{n \mathrm{I}} A_{n}+\frac{1}{a^{n}}{ }^{\mathrm{I}} B_{n}={ }^{y} a_{n}, \quad a^{n \mathrm{R}} A_{n}-\frac{1}{a^{n}}{ }^{\mathrm{R}} B_{n}={ }^{y} b_{n}
\end{array}
$$

These algebraic equations are solved easily for ${ }^{\mathrm{R}} A_{n},{ }^{\mathrm{I}} A_{n},{ }^{\mathrm{R}} B_{n},{ }^{\mathrm{I}} B_{n}$, then coefficients of general mapping function (Eq.9) are determined as follows,

$$
\begin{aligned}
{ }^{\mathrm{R}} A_{n} & =\frac{1}{2 a^{n}}\left({ }^{x} a_{n}+{ }^{y} b_{n}\right),{ }^{\mathrm{R}} B_{n}=\frac{a^{n}}{2}\left({ }^{x} a_{n}-{ }^{y} b_{n}\right) \\
{ }^{\mathrm{I}} A_{n} & =\frac{1}{2 a^{n}}\left({ }^{y} a_{n}-{ }^{x} b_{n}\right),
\end{aligned}
$$

The "disturbance $w$ " is expressed using the complex velocity potential $f$ as follows,

$$
\begin{aligned}
w & =f-U z e^{-i \alpha} \\
& =U\left(\zeta e^{-i \alpha}+\frac{a^{2}}{\zeta} e^{i \alpha}-z e^{-i \alpha}\right)
\end{aligned}
$$

\begin{tabular}{|c|c|c|}
\hline & $\begin{array}{l}\text { General mapping } \\
\text { function, } z\end{array}$ & Mapping diagram \\
\hline Triangle & $\begin{array}{c}0.6288 \zeta_{+} \\
(0.0865 \mathrm{i}) \zeta^{4}- \\
0.000005 \zeta^{5}+\frac{0.0328}{\zeta^{5}}\end{array}$ & \\
\hline Pentagon & $\begin{array}{c}0.8695 \zeta+ \\
(0.0369 \mathrm{i}) \zeta^{6}+ \\
\frac{0.0392 \mathrm{i}}{\zeta^{4}}\end{array}$ & \\
\hline Heptagon & $0.935 \zeta_{-} \frac{0.02 \mathrm{i}}{\tau^{6}}$ & \\
\hline ........ & …..... & \\
\hline
\end{tabular}

Eq.9 is substituted in Eq.12. Then we calculate the virtual mass $M^{\prime}$ as follows,

$$
\begin{aligned}
M^{\prime}=-\frac{1}{2} \frac{i \rho}{U^{2}} & \oint_{c 1} w d \bar{w} \\
=-\rho \pi\{( & \left.1-A_{1}-\bar{A}_{1}\right) a^{2}+A_{1} \bar{A}_{1} a^{2}+2 A_{2} \bar{A}_{2} a^{4}+\cdots \cdots \\
& +n A_{n} \bar{A}_{n} a^{2 n}+\cdots \cdots \\
& -a^{2}+B_{1} e^{-2 i \alpha}+\bar{B}_{1} e^{2 i \alpha}-\frac{B_{1} \bar{B}_{1}}{a^{2}}-\cdots \cdots \\
& \left.-\frac{n B_{n} \bar{B}_{n}}{a^{2 n}}-\cdots \cdots\right\}
\end{aligned}
$$

\section{Results and discussion}

\subsection{Comparison between equilateral polygon}

The comparison amongst different polygons is shown in Table 1 for their respective mapping functions and diagrams.

Table 1. Comparison between equilateral polygons.

In this calculation, number of terms of Laurent series is limited to only four Laurent series coefficients. Therefore descrepancies between 'Geometry (triangle, square,...)' 
and 'Mapping diagram' are appeared. However when more terms are applied to calculate it, it is expected to improve it.

\subsection{Coefficient of virtual mass}

The virtual mass is calculated using exact conformal method[4] so as to make comparison, in which it is more proper for the respective virtual mass to be expressed in the form of coefficient. The coefficient of virtual mass is defined as follows,

$$
C=\frac{M_{\text {body }}}{M_{\text {circumscribed circle }}}
$$

Based on Eq.(14), the coefficient can be obtained by dividing calculated virtual mass of a body with its circumscribed circle. For example, Fig. 2 shows the square and hexagon inscribed in their respective circle.
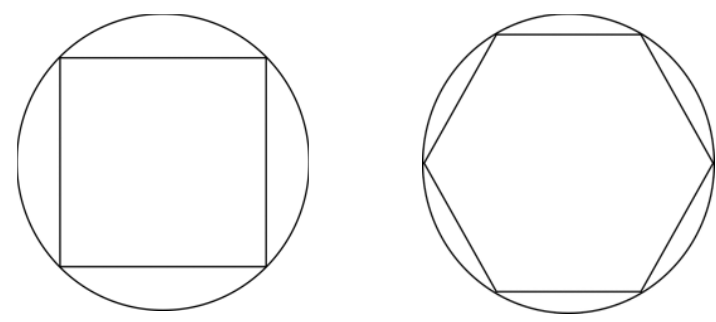

Figure 2. Inscribed square and hexagon.

Table 2 shows the comparison $C_{G}$ (present method) with $C_{E}$ (conformal mapping[4]) .

Table 2. Comparison of virtual mass coefficient for equilateral polygon.

\begin{tabular}{|c|c|c|c|}
\hline & $C_{G}$ (present) & $C_{E}[4]$ & error, $\Delta C$ \\
\hline Triangle & 0.898 & 0.661 & 0.237. \\
\hline Pentagon & 0.981 & 0.833 & 0.148 \\
\hline Heptagon & 0.998 & 0.925 & 0.073 \\
\hline$\ldots \ldots \ldots$ & $\ldots \ldots \ldots$ & $\ldots \ldots \ldots$ & $\ldots \ldots \ldots$ \\
\hline
\end{tabular}

It is shown that the differences decrease as the body shape reaches a circle, in which this is because the coefficient will be equal to unity when it is a circle.

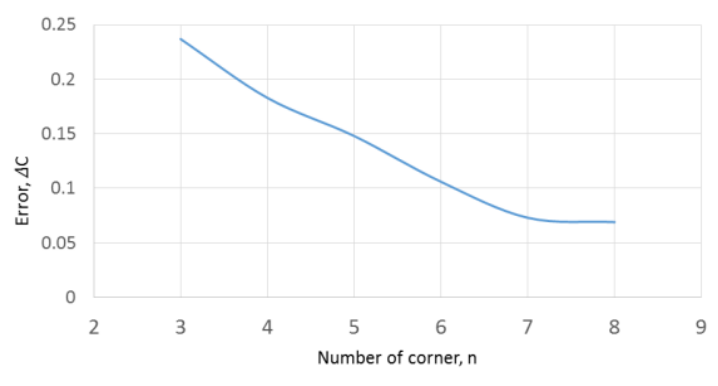

Figure 3. Error.

Figure 3 shows errors which decreases when the body reaches a circle. In this comparison only 4 terms of general mapping function at maximum are used. When much more terms are applied, it is expected that errors are decreasing.

This method is applied for the random shape body. The essence of general mapping method is that it can determine the general mapping function which can be used to map from any shape body in $z$-place to a circle $\zeta$ plane. The concept of Laurent series is the backbone of this newly proposed general mapping method as it can be used complex functions. The general mapping function can be expressed in Eq.(15).

$$
\begin{aligned}
z(\zeta)= & -0.0075+(0.88-0.0604 i) \zeta-0.005 \zeta^{2} \\
& +0.0062 \zeta^{4}-0.069 \zeta^{5}-0.089 \zeta^{6}+\cdots \cdots \\
& +\frac{0.074 i}{\zeta}+\frac{0.005}{\zeta^{2}}+\frac{0.026 i}{\zeta^{3}}+\frac{0.0062}{\zeta^{4}}+\cdots \cdots
\end{aligned}
$$

The general mapping function from Eq.(15) is applied to map the random shape body to a circle shown in Fig.4, for instance.

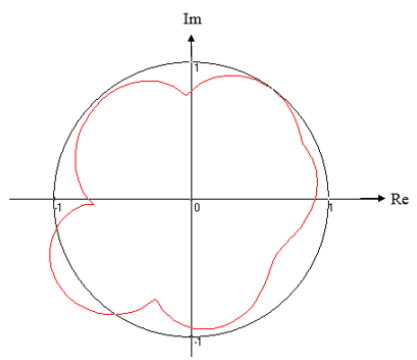

Figure 4. Formation of any shape body with general mapping function.

Note that some coefficients of Eq.(15) are neglected due to very small values which are insignificant to the characteristic of mapping function. 


\section{Conclusion and recommendations}

The analytical solution of virtual mass of any shape body with angle of attack is shown by using complex velocity potential, in which the body shape is expressed by Fourier Series and the conformal mapping function is expressed by Laurent Series. The validation of the analysis is confirmed by comparing with other analytical and exact results (circle, flat plate, regular polygon and so on).

There are some recommendations that are important to be considered in near future in order to improve results.

1. Number of terms of Fourier and Laurent series :In order to obtain the specific general mapping function, Fourier coefficients are required to be determined. In this calculation, number of terms of Fourier series is limited to only five Fourier coefficients for both $a_{n}$ and $b_{n}$. When more coefficients are used to calculate it, it is expected that results are improved to show more accurate results.

2. Convergency of series: In this sample calculation, first 4 or 5 terms of series are applied to calculate the virtual mass. If the convergency is so slow, then more terms should be applied to calculate it. However, when more terms are applied to calculate it, the oscillation llike Gibbs phenomenon may appear. So it is very important to validate the convergency.

\section{References}

1. H. Lamb, Hydrodynamics (Cambridge University Press, $6^{\text {th }}$ ed., 1932)

2. L. M. Milne-Thomson, Theoretical Hydrodynamics (Macmillan, 5th ed., 1968)

3. L. D. Landau and E. M. Lifshitz, Fluid Mechanics (Pergamon Press, 1959)

4. N. Arai, Proc. Of IsySM'2015 International Symposium in Science and Mathematics, Bandung, Indonesia, Nov.24-26, 2015. 